\title{
Recombination between T-DNA insertions to cause chromosomal deletions in Arabidopsis is a rare phenomena
}

\author{
John F Seagrist ${ }^{1}{ }^{\text {, Shih-Heng Su }}{ }^{2}{ }^{\text {, Patrick J Krysan }}{ }^{\text {Corresp. } 1,3}$ \\ 1 Department of Horticulture, University of Wisconsin-Madison, Madison, WI, United States \\ 2 Laboratory of Genetics, University of Wisconsin-Madison, Madison, WI, United States \\ 3 Genome Center of Wisconsin, University of Wisconsin-Madison, Madison, WI, United States \\ Corresponding Author: Patrick J Krysan \\ Email address: pjkrysan@wisc.edu
}

We previously described the identification of a chromosomal deletion in Arabidopsis thaliana that resulted in the elimination of genomic DNA between two T-DNA insertions located ca. 25 kilobases apart on chromosome IV. The mechanism responsible for this deletion appears to have been recombination between the closely spaced T-DNA elements located in trans in a parent plant. In our original study, we observed one such deletion event after screening ca. 2,000 seedlings using a polymerase chain reaction (PCR) assay. Because a method for precisely deleting a selected region of the Arabidopsis genome would have significant value as a research tool, we were interested in determining the frequency with which this type of T-DNA-directed deletion occurs. To do this we designed a genetic screen that would allow us to phenotypically screen for deletions caused by recombination between T-DNA inserts. This screen involved crossing T-DNA single-mutant lines in order to produce $\mathrm{F} 1$ plants in which the two T-DNA insertions flanked a FUSCA (FUS) locus present in the genome. Loss-of-function mutations of FUS genes cause a distinctive developmental phenotype that can be easily scored visually in young seedlings. We used T-DNA lines flanking FUS2, FUS6, FUS7, and FUS11 for this study. Recombination between the T-DNAs in an F1 parent should result in deletion of the FUS gene located between the T-DNAs. Because the deletion would be heterozygous in the F2 generation, we screened the F3 progeny of pooled F2 individuals to search for the fus loss-of-function phenotype. Using this strategy we were able to evaluate a total of 28,314 meioses for evidence of deletions caused by recombination between the T-DNA inserts. No seedlings displaying the fus phenotype were recovered, suggesting that deletions caused by recombination between T-DNA inserts are relatively rare events and may not be a useful tools for genome engineering in Arabidopsis. 
1

2

3

4 Recombination between T-DNA insertions to cause chromosomal deletions in Arabidopsis

5 is a rare phenomena

6

7

8

9 John Seagrist ${ }^{1}$, Shih-Heng $\mathrm{Su}^{2}$, and Patrick J Krysan ${ }^{1,3}$

10

11

12

13 14

15

16

17 18

19

20

21

22

23

24

25

26

27

28

29

30

${ }^{1}$ Department of Horticulture, University of Wisconsin-Madison, Madison, WI, USA 4

${ }^{2}$ Laboratory of Genetics, University of Wisconsin-Madison, Madison, WI, USA

7

${ }^{3}$ Genome Center of Wisconsin, University of Wisconsin-Madison, Madison, WI, USA

8

9

0

1

22

Corresponding author: Patrick J Krysan

3

pjkrysan@wisc.edu

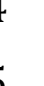

7

8

9 
31 ABSTRACT

32 We previously described the identification of a chromosomal deletion in Arabidopsis

33 thaliana that resulted in the elimination of genomic DNA between two T-DNA insertions

34 located ca. 25 kilobases apart on chromosome IV. The mechanism responsible for this

35 deletion appears to have been recombination between the closely spaced T-DNA elements

36 located in trans in a parent plant. In our original study, we observed one such deletion

37 event after screening ca. 2,000 seedlings using a polymerase chain reaction (PCR) assay.

38 Because a method for precisely deleting a selected region of the Arabidopsis genome would

39 have significant value as a research tool, we were interested in determining the frequency

40 with which this type of T-DNA-directed deletion occurs. To do this we designed a genetic

41 screen that would allow us to phenotypically screen for deletions caused by recombination

42 between T-DNA inserts. This screen involved crossing T-DNA single-mutant lines in order

43 to produce F1 plants in which the two T-DNA insertions flanked a FUSCA (FUS) locus

44 present in the genome. Loss-of-function mutations of FUS genes cause a distinctive

45 developmental phenotype that can be easily scored visually in young seedlings. We used T-

46 DNA lines flanking FUS2, FUS6, FUS7, and FUS11 for this study. Recombination between the

47 T-DNAs in an F1 parent should result in deletion of the FUS gene located between the T-

48 DNAs. Because the deletion would be heterozygous in the F2 generation, we screened the

49 F3 progeny of pooled F2 individuals to search for the fus loss-of-function phenotype. Using

50 this strategy we were able to evaluate a total of 28,314 meioses for evidence of deletions

51 caused by recombination between the T-DNA inserts. No seedlings displaying the fus

52 phenotype were recovered, suggesting that deletions caused by recombination between T-

53 DNA inserts are relatively rare events and may not be a useful tools for genome

54 engineering in Arabidopsis. 


\section{INTRODUCTION}

57 In order to understand the function of a genome, it is critical to have tools available for

58 modifying the DNA sequence of that genome. In the case of the model plant Arabidopsis

59 thaliana, one of the most widely used strategies for knocking out a gene of interest is

60 insertional mutagenesis based on the transferred DNA (T-DNA) of Agrobacterium

61 tumefaciens (Alonso et al. 2003; Krysan et al. 1999; O'Malley \& Ecker 2010; Parinov \&

62 Sundaresan 2000). A number of labs throughout the world have produced large

63 populations of Arabidopsis transgenic lines in which T-DNA elements are randomly

64 inserted throughout the genome (Alonso et al. 2003; Brunaud et al. 2002; Ito et al. 2002;

65 Rosso et al. 2003; Sessions et al. 2002; Woody et al. 2007). The exact positions of the T-

66 DNAs are determined by DNA sequencing (Alonso et al. 2003). Currently, there are over

67400,000 T-DNA insertion lines cataloged in the Arabidopsis genome, with the

68 corresponding seed available through public stock centers. Although the T-DNA elements are inserted "randomly", the existence of such a large catalog of lines means that most of the genes in Arabidopsis have at least one T-DNA mutant allele available. These public TDNA collections are an extremely popular community resource for studying gene function in Arabidopsis. Because approximately 17\% of the genes in Arabidopsis are members of a tandemly-duplicated gene family, however, it can be challenging to study gene function using T-DNA knockouts when the gene of interest has a second copy nearby on the same chromosome (Initiative 2000). If these duplicated genes display functional redundancy, one must knockout both genes in order to reveal a mutant phenotype. Because of the extremely tight genetic linkage between tandemly duplicated genes, it is not practical to rely on meiotic recombination to produce a double-mutant for tandemly duplicated genes using T-DNA insertion lines.

The Arabidopsis MAP kinase kinase kinase gene $M E K K 1$ is part of a tandemly duplicated gene family in which $M E K K 1, M E K K 2$, and $M E K K 3$ are located within a ca. 25 kilobase $(\mathrm{kb})$ region of chromosome IV. In a previous study we reported the identification of a deletion mutant that appeared to be the result of recombination between a T-DNA insertion in MEKK1 and a second T-DNA located ca. $21 \mathrm{~kb}$ away in $M E K K 3$ (Su et al. 2013). Because the two T-DNA insertions were produced using the same vector, meiotic 
87 been responsible for producing the deletion of the intervening genomic DNA. This deletion

88 mutant proved to be valuable for dissecting the function of the $M E K K 1 / 2 / 3$ gene family (Su 89 et al. 2013).

90 Due to the prevalence of tandemly-duplicated gene families in Arabidopsis, we were 91 interested in determining if deletions caused by recombination between T-DNA inserts 92 might be useful tools for performing genome engineering in Arabidopsis. In our study of 93 the $M E K K 1 / 2 / 3$ tandem gene family, we identified the deletion mutant using a PCR-based 94 screen of ca. 2,000 seedlings. Since we only found one individual carrying the deletion, it 95 was not possible to accurately estimate the frequency with which this type of T-DNA 96 directed deletion might occur. In order for deletions caused by recombination between TDNA insertions to be useful genome engineering tools, the frequency with which it occurs needs to be within a practical range to make screening for deletions cost- and timeeffective. We therefore designed a genetic screen that enabled us to phenotypically screen for deletions caused by recombination between T-DNA inserts at a number of locations in

101

102

103

104

105

106

107

108

109

110

111

112

113

114

115

116 117 the Arabidopsis genome. Despite screening 28,314 meioses for evidence of T-DNA-directed deletions, none were found. These results suggest that deletions caused by recombination between T-DNA inserts may be relatively rare events and that their utility for genome engineering may be limited.

\section{MATERIALS AND METHODS}

\section{Plant Material and Growth Conditions}

Arabidopsis lines carrying T-DNA insertions flanking FUS2 (At4g10180), FUS6 (At3g61140), Fus7 (At4g14110), and Fus11 (At5g14250) were obtained from the Arabidopsis Biological Resource Center at The Ohio State University (abrc.osu.edu). FUS2: SALK_046021 and SALK_004560. FUS6: WiscDsLox453-456F5, WiscDsLox402F03, SALK_203944, and SALK_062171. FUS7: SALK_073580 and SALK_150313. FUS11: SALK_150318 and SALK_150319. Seedlings were germinated on $1 \%$ agar (w/v) plates containing $0.5 \times$ Murashige and Skoog (MS) basal salt mixture under continuous light at $20^{\circ}-23^{\circ} \mathrm{C}$. Seedlings were transferred to a $2: 1$ mixture (vol:vol) of potting mix:pearlite 5 to 7 days after germination and grown under constant light at $20^{\circ}-23^{\circ} \mathrm{C}$. 


\section{Genotyping}

119 PCR-based genotyping was used to determine the presence of the desired T-DNA insertion

120 elements. Genomic DNA was extracted from $5 \mathrm{~mm}$ square pieces of leaf tissue as previously

121 described (Kasajima et al. 2004). PCR was performed using the gene-specific PCR primers

122 indicated in Table 1 in conjunction with the T-DNA left border primer p745: 5'-

123 AACGTCCGCAATGTGTTATTAAGTTGTC-3'. The thermal cycling conditions used were as

124 follows: $96^{\circ} \mathrm{C}$ for 2 minutes, followed by 40 cycles of $94^{\circ} \mathrm{C}$ for 10 seconds, $58^{\circ} \mathrm{C}$ for 30

125 seconds, and $72^{\circ} \mathrm{C}$ for 30 seconds. PCR products were visualized by agarose gel

126 electrophoresis followed by ethidium bromide staining.

127

\section{Phenotypic Screening}

129 F1 plants were produced by crossing two single-mutant T-DNA lines. PCR genotyping was

130 used to confirm that the F1 plants carried the desired T-DNA insertions. F2 seed was then

131 collected from individual F1 plants. F2 seedlings were germinated on petri dishes

132 containing agar growth media and transplanted to potting mix at a density of 9 seedlings

133 per 3.5 inch square pot. The F2 plants were allowed to self-pollinate, and the resulting

134 seed was collected in bulk from each pot of 9 plants. Ca. 1,200 F3 seeds from each pool of

135 nine F2 plants were placed onto water-saturated $125 \mathrm{~mm}$ diameter circular pieces of

136 Whatman filter paper (Whatman cat. \#1004-125) inside 140mm diameter plastic petri

137 dishes. The plates were then incubated in the dark at $4^{\circ} \mathrm{C}$ for 2 to 3 days to stratify the

138 seeds. The plates were then placed under light at $20^{\circ}-23^{\circ} \mathrm{C}$ for four hours to stimulate

139 germination, and then transferred to black box with no light at $20^{\circ}-23^{\circ} \mathrm{C}$ for four days to

140 allow for etiolated growth. Plates were then visually inspected to search for seedlings

141 displaying the distinctive fus phenotype.

142

143

144 RESULTS

145 Design of the Genetic Screen

146 We use the term "deletion caused by T-DNA recombination" to refer to the situation where

147 meiotic crossing over between T-DNA insertions located nearby to each other leads to

148 deletion of the intervening genomic DNA (Fig. 1). In our previous study describing the 
149 identification of a deletion caused by T-DNA recombination in the $M E K K 1 / 2 / 3$ gene family

150 we used a PCR-based method to identify a single deletion mutant after screening ca. 2,000

151 seedlings (Su et al. 2013). In the present study, our objective was to screen a larger

152 population of individuals in order to better ascertain the frequency with which deletions

153 caused by T-DNA recombination occur. In order to do this in a cost-effective manner, we

154 chose to develop a genetic screen that would let us visually screen for evidence of deletions

155 caused by T-DNA recombination. The rationale for the screen was to choose loci in the

156 Arabidopsis genome for which null mutants produced a striking visual phenotype that

157 could be easily scored at the early seedling stage. We chose to use FUSCA (FUS) genes for

158 this purpose (Misera et al. 1994). The FUS genes are negative regulators of

159 photomorphogenesis such that null mutants cause dark-grown seedlings to display

160 characteristics normally seen in light-grown seedlings. When Arabidopsis seeds are

161 germinated and grown in the dark for four days, the hypocotyls elongate, the apical hook

162 remains closed, and the seedlings are pale white in appearance. By contrast, fus mutant

163 seedlings grown in the dark have short hypocotyls, the apical hook is open, and they are

164 dark purple due to anthocyanin accumulation (Misera et al. 1994). A fus mutant can

165 therefore be easily distinguished on a plate of thousands of wild-type dark grown seedlings

166 as a short purple seedling with open cotyledons against a background of long, skinny, white

167 seedlings. This made the fus mutant phenotype a good candidate for our genetic screen.

168 There are at least fourteen FUS loci (Misera et al. 1994) in the genome, and these genes

169 have also been identified as CONSTITUTIVE PHOTOMORPHOGENIC (COP) and DE-

170 ETIOALTED (DET) (Huang et al. 2014).

171 To identify specific FUS loci for our screen, we searched the available T-DNA mutant

172 collections for FUS genes that had T-DNA insertions in close proximity upstream and

173 downstream of the FUS gene. Suitable T-DNA lines were found for FUS2, FUS6, FUS7, and

174 FUS11 (Fig. 2). In the case of FUS6, we chose two different sets of T-DNA lines flanking

175 FUS6, one set from the SALK T-DNA line collection (Alonso et al. 2003) and the other from

176 the WiscDsLox collection (Woody et al. 2007). The T-DNA lines for FUS2, FUS7, and FUS11

177 were from the SALK collection.

178 In our previous study involving deletion of the $M E K K 1 / 2 / 3$ locus, we determined that

179 T-DNA left border sequences were present on both sides of the T-DNA inserts for MEKK1 
180 and MEKK3. This observation suggested that the at least two copies of the T-DNA

181 monomer were inserted at each locus in an inverted repeat orientation. Since inverted

182 repeats have the potential to form hairpin structures that may have the ability to stimulate

183 recombination, we were interested in determining if some of the T-DNA inserts used in the

184 present study had left border sequences on both sides of the insert. PCR reactions using

185 left-border specific primers and gene specific primers were used to map the left-border

186 sequences present at each locus. As shown in Figure 2, at least one of the T-DNA inserts in

187 each pair had left border sequence on both sides of the insert. In the case of FUS7 and

188 FUS11, both the upstream and downstream T-DNA inserts had left border sequences on 189 both sides.

190 For a given FUS gene, the upstream and downstream T-DNA lines were crossed 191 together to produce F1 plants carrying two T-DNA insertions: one upstream of the FUS

192 gene and one downstream (Fig. 1). If recombination occurs between those two T-DNA

193 insertions during meiosis, one would expect one of the meiotic products to carry a deletion

194 of the FUS gene. Unless both the pollen and egg carried deletion events, one would expect

195 T-DNA directed deletions produced by an F1 plant to be heterozygous in the F2 progeny

196 (Fig. 1). Since the fus phenotype is a recessive condition, it is necessary to perform F3

197 progeny testing to identify the presence of a T-DNA directed deletion carried by an F2

198 plant. To streamline this process, we grew $9 \mathrm{~F} 2$ plants together in a single pot and

199 collected their F3 progeny in bulk. Ca. 1,200 F3 seeds from each pot were then germinated

200 in the dark on moist filter paper and visually scored after four days to screen for the fus

201 phenotype. This represents an average of 133 progeny for each F2 parent. If any of the F2 202 parents were carrying a heterozygous deletion of the FUS gene, then we would expect $25 \%$ 203 of those progeny, or ca. 33 seedlings, to display the fus phenotype. In dark growth 204 conditions, fus seedlings are short and purple with open cotyledons, which would stand in 205 stark contrast to the long, white, etiolated form of wild-type seedlings.

206

\section{Results of Genetic Screen}

208 F3 progeny collected from a total of 14,157 F2 parent plants were screened for the fus 209 phenotype as described above in order to find seedlings potentially carrying deletions 210 caused by T-DNA recombination of the FUS gene (Table 2). Since the recombination event 
211 that would lead to a deletion could occur during meiosis in either the male of female

212 gametophyte, the population of 14,157 F2 plants that we screened represented 28, 314

213 meioses. The distribution of these F2 plants between the different FUS loci used in the

214 study are shown in Table 2. No fus mutant seedlings were observed in any of the F3

215 progeny tested.

216

217 DISCUSSION

218 The objective of this study was to determine the rate with which recombination occurs

219 between T-DNA inserts located nearby to each other in the Arabidopsis genome. One of the

220 consequences of this type of recombination is the deletion of chromosomal DNA located

221 between the two T-DNA inserts. We use the term "deletions caused by T-DNA

222 recombination" to describe this phenomena. This type of deletion is caused by

223 recombination between two established T-DNA inserts that were generated via

224 independent transformation events in separate plants. Recently, it has been shown that

225 during the initial T-DNA integration process that heterogeneous T-DNA integration can

226 cause chromosomal rearrangements, including deletions, as part of the double-stranded

227 break repair mechanism driving T-DNA insertion (Hu et al. 2017). This type of T-DNA

228 associated deletion is mechanistically distinct from the phenomena that we were targeting 229 with our study.

230 In our previous work we observed one instance of a deletion caused by T-DNA 231 recombination in a population of ca. 2,000 seedlings (Su et al. 2013). Because of the 232 extensive collections of Arabidopsis T-DNA insertion lines available through public stock

233 centers, we were intrigued by the possibility that deletions caused by T-DNA

234 recombination could provide an effective strategy for genome engineering. If the rate of

235 deletion caused by T-DNA recombination was high enough, one could imagine obtaining a

236 pair of T-DNA lines flanking a genomic region of interest, producing an F1 parent carrying

237 those two T-DNA insertions, and then screening for deletions in the F2 progeny. Since the 238 genetic screen described in this study did not reveal any evidence for deletions caused by

239 T-DNA recombination from a population of 14,157 F2 parent plants, it appears that this

240 type of deletion may be a relatively rare event. 
241 The most straightforward interpretation of our failure to observe fus mutant

242 seedlings in our genetic screen is that deletions caused by T-DNA recombination did not

243 occur in the F1 plants used in our study. It is also possible, however, that deletions may

244 have been generated, but were subsequently selected against due to lethality or reduced

245 fitness of the specific deletion allele that was formed. In most of the FUS loci used in our

246 study, the upstream and downstream T-DNA inserts are located in neighboring genes. It is

247 possible that deleting those neighboring genes in addition to the FUS gene could have a

248 detrimental effect on the plant. It should be noted that each of the T-DNA lines used for this

249 study was viable when the T-DNA insert was homozygous, indicating that the neighboring

250 genes are not essential genes and that deleting them on their own should not cause

251 lethality. It nevertheless remains possible that genetic interactions between the loci or

252 other functional elements contained within the intervals between the flanking T-DNAs

253 could lead to unexpected lethality of the predicted deletions.

254

\section{CONSLUSIONS}

256 Our study has suggested that the deletion caused by T-DNA recombination

257 phenomena is unlikely to be a useful strategy for genome engineering in Arabidopsis. Given

258 recent advances in the application of CRISPR-Cas9 methods in plants, a more effective

259 approach may be to direct double-strand breaks using Cas 9 targeted to sites flanking the

260 region of interest (Yin et al. 2017). This type of approach has been used in rice to delete

261 regions up to $245 \mathrm{~kb}$ in size (Zhou et al. 2014) and Arabidopsis to delete regions up to 120

$262 \mathrm{~kb}$ (Ordon et al. 2017). Despite the lack of an immediate practical application, our previous

263 observation of recombination between established T-DNA insertions highlights the

264 dynamic nature of genome structure. As our ability to modify genomes advances, there

265 remains a need to more fully understand all of the mechanisms and processes that shape

266 genome structure and behavior.

267

268

269

270

LITERATURE CITED

271 
272

273

274

275

276

277

278

279

280

281

282

283

284

285

286

287

288

289

290

291

292

293

294

295

296

297

298

299

300

301

302

303

304

305

306

307

308

309

310

311

312

313

314

315

316

Alonso JM, Stepanova AN, Leisse TJ, Kim CJ, Chen H, Shinn P, Stevenson DK, Zimmerman J, Barajas P, Cheuk R, Gadrinab C, Heller C, Jeske A, Koesema E, Meyers CC, Parker H, Prednis L, Ansari Y, Choy N, Deen H, Geralt M, Hazari N, Hom E, Karnes M, Mulholland C, Ndubaku R, Schmidt I, Guzman P, Aguilar-Henonin L, Schmid M, Weigel D, Carter DE, Marchand T, Risseeuw E, Brogden D, Zeko A, Crosby WL, Berry CC, and Ecker JR. 2003. Genome-wide insertional mutagenesis of Arabidopsis thaliana. Science 301:653-657. 10.1126/science.1086391

301/5633/653 [pii]

Brunaud V, Balzergue S, Dubreucq B, Aubourg S, Samson F, Chauvin S, Bechtold N, Cruaud C, DeRose R, Pelletier G, Lepiniec L, Caboche M, and Lecharny A. 2002. T-DNA integration into the Arabidopsis genome depends on sequences of pre-insertion sites. EMBO Rep 3:1152-1157. 10.1093/embo-reports/kvf237

Hu Y, Chen Z, Zhuang C, and Huang J. 2017. Cascade of chromosomal rearrangements caused by a heterogeneous T - DNA integration supports the double - stranded break repair model for T - DNA integration. The Plant Journal 90:954-965. doi:10.1111/tpj.13523

Huang X, Ouyang X, and Deng XW. 2014. Beyond repression of photomorphogenesis: role switching of COP/DET/FUS in light signaling. Curr Opin Plant Biol 21:96-103. 10.1016/j.pbi.2014.07.003

Initiative AG. 2000. Analysis of the genome sequence of the flowering plant Arabidopsis thaliana. Nature 408:796-815. 10.1038/35048692

Ito T, Motohashi R, Kuromori T, Mizukado S, Sakurai T, Kanahara H, Seki M, and Shinozaki K. 2002. A new resource of locally transposed Dissociation elements for screening gene-knockout lines in silico on the Arabidopsis genome. Plant Physiol 129:16951699. 10.1104/pp.002774

Kasajima I, Ide Y, Ohkama-Ohtsu N, Hayashi H, Yoneyama T, and Fujiwara T. 2004. A Protocol for Rapid DNA Extraction From Arabidopsis thaliana for PCR Analysis. Plant Molecular Biology Reporter 22:49-52.

Krysan PJ, Young JC, and Sussman MR. 1999. T-DNA as an insertional mutagen in Arabidopsis. Plant Cell 11:2283-2290.

Misera S, Muller AJ, Weiland-Heidecker U, and Jurgens G. 1994. The FUSCA genes of Arabidopsis: negative regulators of light responses. Mol Gen Genet 244:242-252.

O'Malley RC, and Ecker JR. 2010. Linking genotype to phenotype using the Arabidopsis unimutant collection. Plant J 61:928-940. 10.1111/j.1365-313X.2010.04119.x

TPJ4119 [pii]

Ordon J, Gantner J, Kemna J, Schwalgun L, Reschke M, Streubel J, Boch J, and Stuttmann J. 2017. Generation of chromosomal deletions in dicotyledonous plants employing a user-friendly genome editing toolkit. Plant J 89:155-168. 10.1111/tpj.13319

Parinov S, and Sundaresan V. 2000. Functional genomics in Arabidopsis: large-scale insertional mutagenesis complements the genome sequencing project. Curr Opin Biotechnol 11:157-161. S0958-1669(00)00075-6 [pii]

Rosso MG, Li Y, Strizhov N, Reiss B, Dekker K, and Weisshaar B. 2003. An Arabidopsis thaliana T-DNA mutagenized population (GABI-Kat) for flanking sequence tag-based reverse genetics. Plant Mol Biol 53:247-259. 10.1023/B:PLAN.0000009297.37235.4a 
317 Sessions A, Burke E, Presting G, Aux G, McElver J, Patton D, Dietrich B, Ho P, Bacwaden J, Ko C, Clarke JD, Cotton D, Bullis D, Snell J, Miguel T, Hutchison D, Kimmerly B, Mitzel T, Katagiri F, Glazebrook J, Law M, and Goff SA. 2002. A high-throughput Arabidopsis reverse genetics system. Plant Cell 14:2985-2994.

Su SH, Bush SM, Zaman N, Stecker K, Sussman MR, and Krysan P. 2013. Deletion of a tandem gene family in Arabidopsis: increased MEKK2 abundance triggers autoimmunity when the MEKK1-MKK1/2-MPK4 signaling cascade is disrupted. Plant Cell 25:1895-1910. 10.1105/tpc.113.112102

Woody ST, Austin-Phillips S, Amasino RM, and Krysan PJ. 2007. The WiscDsLox T-DNA collection: an arabidopsis community resource generated by using an improved high-throughput T-DNA sequencing pipeline. J Plant Res 120:157-165. 10.1007/s10265-006-0048-x

Yin K, Gao C, and Qiu JL. 2017. Progress and prospects in plant genome editing. Nat Plants 3:17107. 10.1038/nplants.2017.107

Zhou H, Liu B, Weeks DP, Spalding MH, and Yang B. 2014. Large chromosomal deletions and heritable small genetic changes induced by CRISPR/Cas9 in rice. Nucleic Acids Res 42:10903-10914. 10.1093/nar/gku806 
Figure 1 (on next page)

Schematic of T-DNA directed deletion and genetic screen.

(A) Recombination between the T-DNA insertions results in deletion of the fus gene. (B) A TDNA directed deletion that occurs during meiosis in an F1 parent will not produce a visible fus phenotype until the F3 generation 
A.

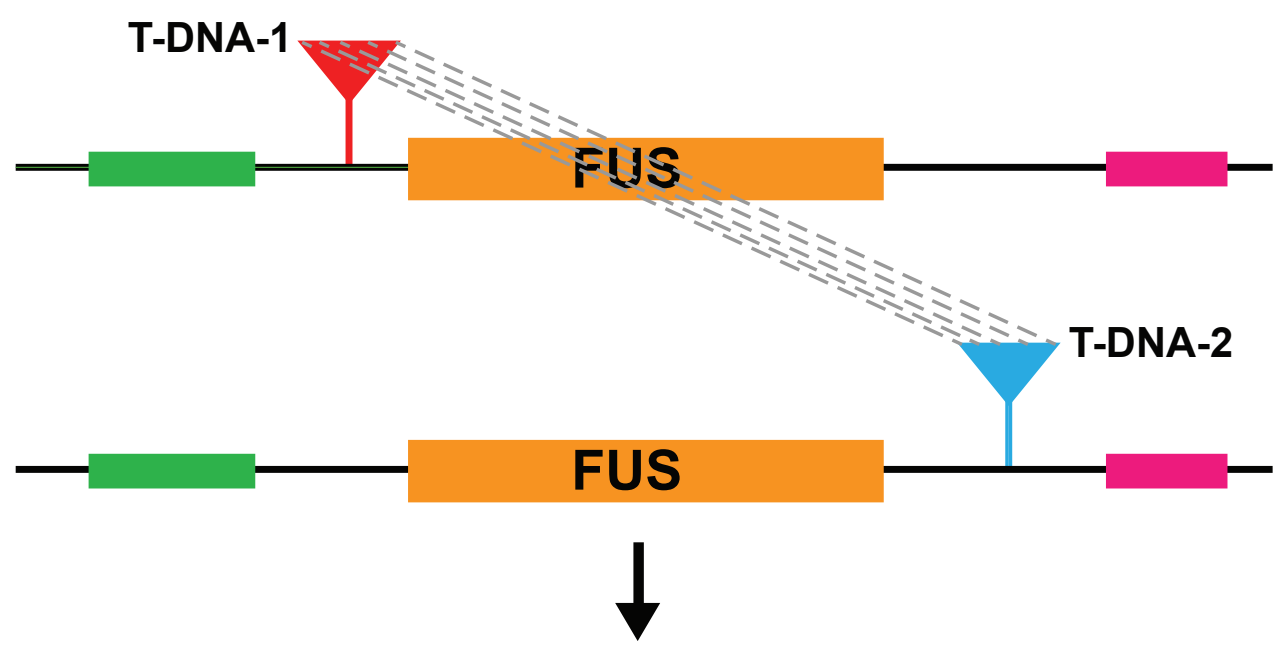

Crossing over between T-DNA-1 and T-DNA-2 leads to deletion of chromsomal DNA on one of the recombined chromosomes

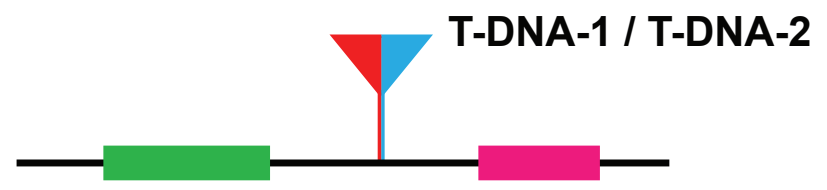

B.

\begin{tabular}{|c|c|c|c|c|c|c|}
\hline F1 Generation & $\rightarrow$ & F2 Generation & $\rightarrow$ & F3 Generat & ion & \\
\hline${ }_{\text {T-DNA-1 }}||_{\text {T-DNA-2 }}$ & & ${ }_{\text {T-DNA-1 }} \mid<\Delta$ & & $\|_{k}$ & $\|$ & \\
\hline $\begin{array}{l}\text { T-DNA-1 line crossed } \\
\text { with T-DNA-2 line }\end{array}$ & & $\begin{array}{c}\text { Deletion } \Delta \\
\text { Heterozygous }\end{array}$ & & $\begin{array}{c}\text { Deletion } \\
\text { Homozygous }\end{array}$ & $\begin{array}{l}\text { Hetero- } \\
\text { zygous }\end{array}$ & $\begin{array}{c}\text { T-DNA-1 } \\
\text { Homozygous }\end{array}$ \\
\hline
\end{tabular}


Figure 2 (on next page)

Maps of FUS loci used in this genetic screen.

Positions of the T-DNA inserts used for each FUS locus are indicated with blue triangles. The presence of the T-DNA Left Border sequence is indicated by "LB". "LB" on both sides of the TDNA suggests that there are at least two copies of the T-DNA element inserted in an inverted repeat orientation. All maps are drawn to the same scale as indicated by the scale bar. 

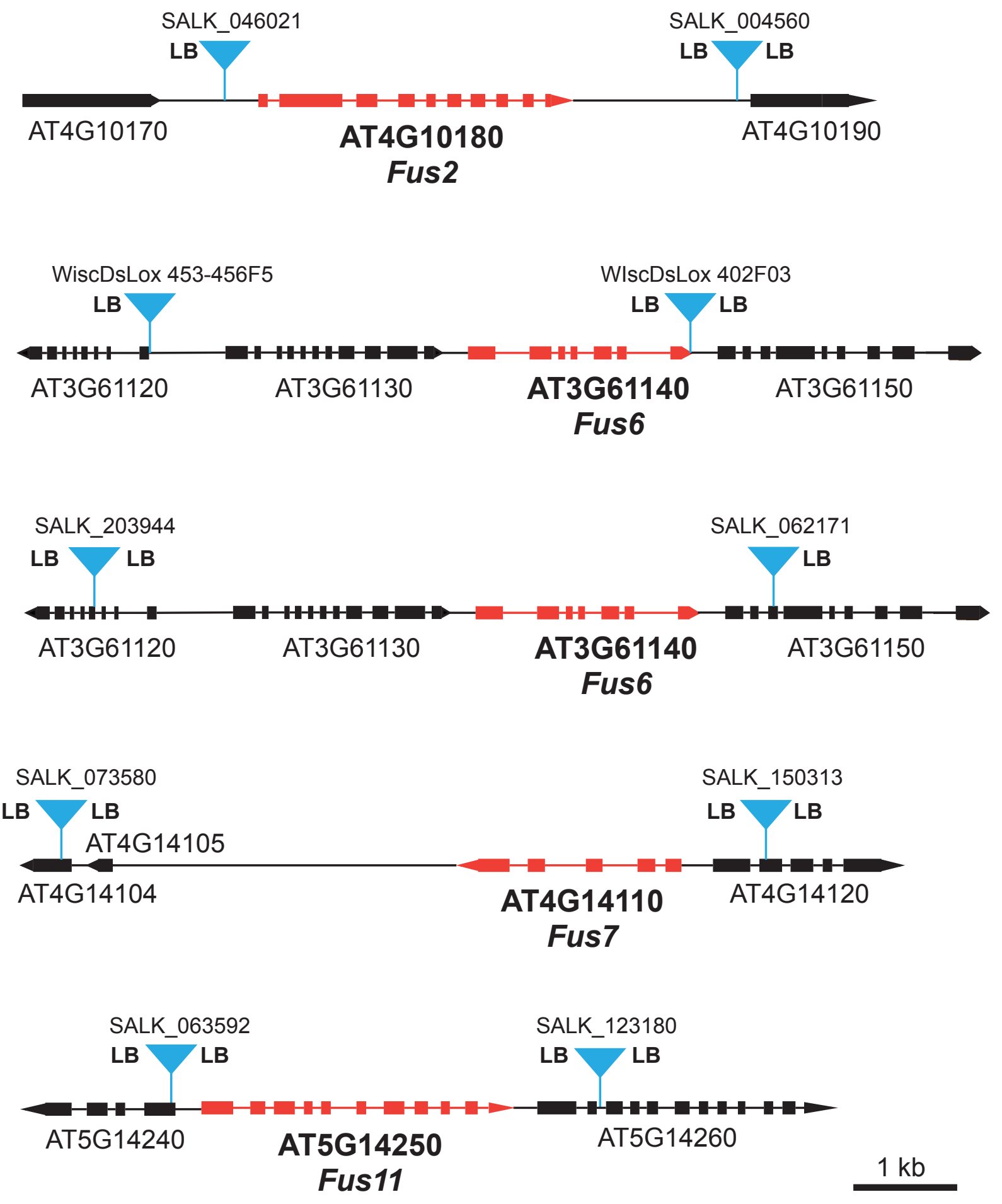
Table $\mathbf{1}$ (on next page)

PCR Primers 


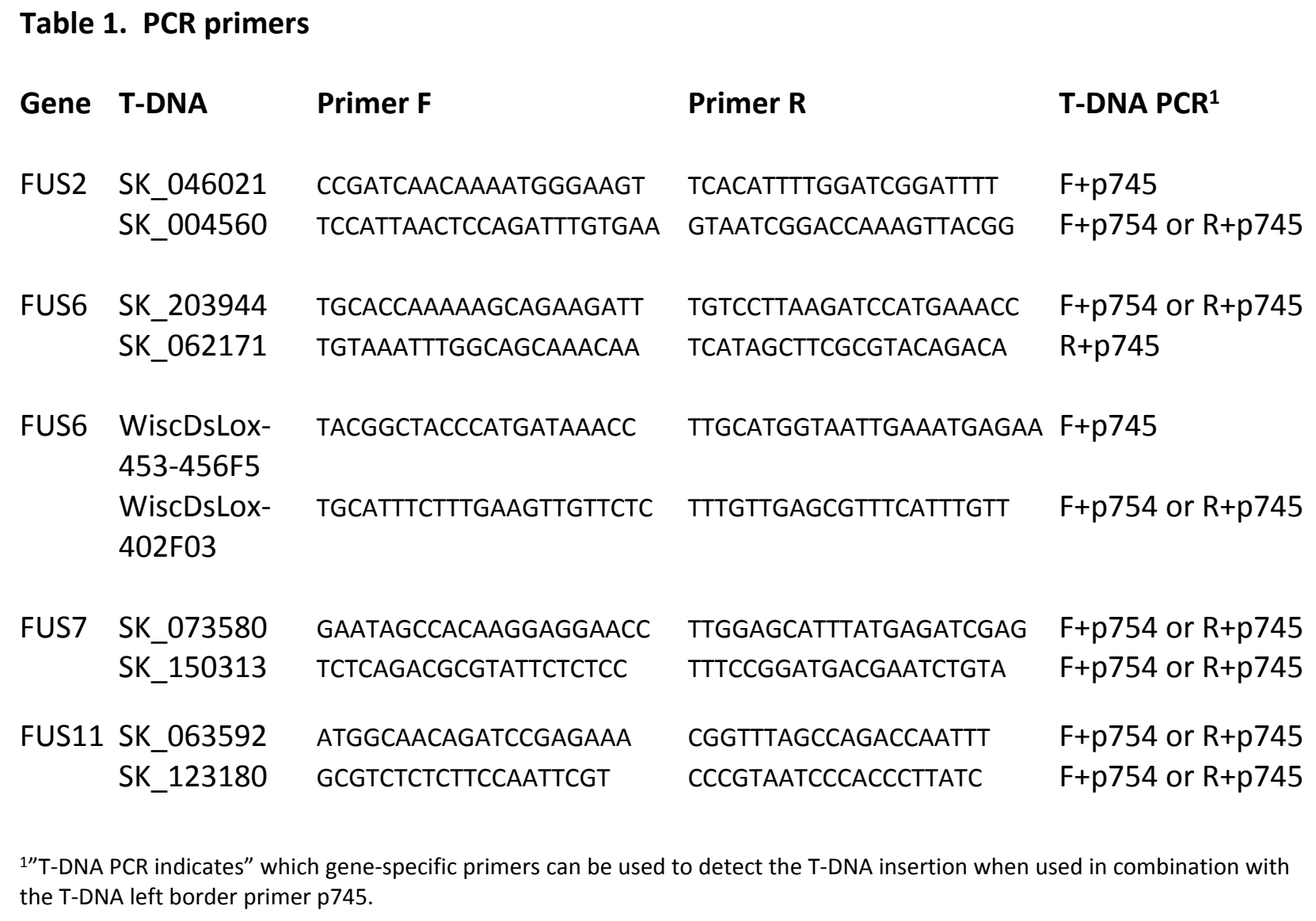

Primer R

FUS2 SK_046021 SK_004560

CCGATCAACAAAATGGGAAGT

TCCATTAACTCCAGATTTGTGAA

TGCACCAAAAAGCAGAAGATT

TGTAAATTTGGCAGCAAACAA

SK 062171

FUS6 WiscDsLox453-456F5

WiscDsLox-

TACGGCTACCCATGATAAACC

TCACATTTTGGATCGGATTTT

GTAATCGGACCAAAGTTACGG

TGTCCTTAAGATCCATGAAACC

TCATAGCTTCGCGTACAGACA 402F03

FUS7 SK_073580

SK_150313

GAATAGCCACAAGGAGGAACC

TTGGAGCATTTATGAGATCGAG

TCTCAGACGCGTATTCTCTCC

TTTCCGGATGACGAATCTGTA

$F+p 754$ or $R+p 745$

FUS11 SK_063592

ATGGCAACAGATCCGAGAAA

CGGTTTAGCCAGACCAATTT

CCCGTAATCCCACCCTTATC

$F+p 754$ or $R+p 745$

GCGTCTCTCTTCCAATTCGT

CCCGTAATCCCACCCTTATC GCGTCTCTCTTCCAATTCGT

1"T-DNA PCR indicates" which gene-specific primers can be used to detect the T-DNA insertion when used in combination with the T-DNA left border primer $\mathrm{p} 745$. 
Table 2 (on next page)

Genetic Screen Results 


\begin{tabular}{|c|c|c|c|c|}
\hline Gene & T-DNA 1 & T-DNA 2 & \# of F2 Parents & \# of Deletions \\
\hline FUS2 & SK_046021 & SK_004560 & 2,916 & 0 \\
\hline FUS6 & SK_203944 & SK_062171 & 2,592 & 0 \\
\hline FUS6 & WiscDsLox 453-456F5 & WiscDsLox $402 \mathrm{F03}$ & 3,312 & 0 \\
\hline FUS7 & SK_073580 & SK_150313 & 2,583 & 0 \\
\hline FUS11 & SK_063592 & SK_123180 & 2,754 & 0 \\
\hline Total & & & 14,157 & 0 \\
\hline
\end{tabular}

